\title{
Retardo na chegada da pessoa com acidente vascular cerebral a um serviço hospitalar de referência
}

RESUMO | Objetivos: Analisar fatores que retardam o atendimento dos pacientes na fase aguda do Acidente Vascular Cerebral em um hospital público de referência. Métodos: Estudo de corte transversal, realizado na cidade de Salvador/Bahia com 50 pacientes através de dados em prontuários e por entrevista semiestruturada. Os dados foram analisados através do programa SPSS versão 21, utilizado o Qui-quadrado para as variáveis do tempo, com nível de significância de 5\%. Resultados: Evidenciouse média de idade de 61,2 \pm 13 anos, predominando o sexo masculino. A maioria dos pacientes não realizou trombólise devido a fatores de atraso como: utilização de carro próprio, não ter um familiar presente na hora do evento, chegada ao hospital fora da janela terapêutica e procura por outros serviços anteriormente. Conclusão: Diversos fatores retardam o atendimento ao paciente com Acidente Vascular Cerebral. Desta maneira, observa-se a necessidade de melhorias no atendimento associada à difusão de informações à população.

Palavras-chaves: Acidente Vascular Cerebral; Serviços Médicos de Emergência; Terapia Trombolítica; Acesso aos Serviços de Saúde.

ABSTRACT | Objectives: To analyze factors that delay the care of patients in the acute phase of stroke in a public referral hospital. Methods: Cross-sectional study, conducted in the city of Salvador / Bahia with 50 patients using data from medical records and semi-structured interviews. The data were analyzed using the SPSS version 21 program, using the Chi-square for the time variables, with a significance level of $5 \%$. Results: There was a mean age of $61.2 \pm 13$ years, with a predominance of males. Most patients did not undergo thrombolysis due to delay factors such as: using their own car, not having a family member present at the time of the event, arriving at the hospital outside the therapeutic window and looking for other services previously. Conclusion: Several factors delay the care of patients with stroke. Thus, there is a need for improvements in care associated with the dissemination of information to the population.

Keywords: Stroke, Emergency Medical Services, Thrombolytic Therapy, Access to Health Services.

RESUMEN | Objetivos: Analizar los factores que retrasan la atención de pacientes en fase aguda de ictus en un hospital público de referencia. Métodos: Estudio transversal, realizado en la ciudad de Salvador / Bahía con 50 pacientes utilizando datos de historias clínicas y entrevistas semiestructuradas. Los datos se analizaron mediante el programa SPSS versión 21, utilizando el Chi-cuadrado para las variables de tiempo, con un nivel de significancia del 5\%. Resultados: la edad media fue de $61,2 \pm 13$ años, con predominio del sexo masculino. La mayoría de los pacientes no se sometieron a trombólisis por factores de demora como: utilizar su propio coche, no tener un familiar presente en el momento del evento, llegar al hospital fuera de la ventana terapéutica y buscar otros servicios previamente. Conclusión: varios factores retrasan la atención de los pacientes con ictus. Por tanto, existe la necesidad de mejoras en la atención asociadas a la difusión de información a la población.

Palabras claves: Accidente cerebrovascular, servicios médicos de emergencia, terapia trombolítica, acceso a servicios de salud.

\section{Paloma de Castro Brandão}

Enfermeira. Doutoranda em Saúde Coletiva pelo Instituto de Saúde Coletiva/UFBA. Mestra em Saúde Coletiva pelo Instituto Sírio Libanês de Ensino e Pesquisa. Especialista em Enfermagem Intensivista. Escola de Enfermagem/UFBA.

ORCID: 0000-0002-8659-6292

\section{Mariana Oliveira Antunes Ferraz}

Enfermeira. Doutoranda em Enfermagem e Saúde pelo Programa de Pós-graduação em Enfermagem e Saúde/UFBA. Mestra em Ciências da Saúde pelo Programa de Pós-graduação em Enfermagem em Saúde/UESB. Universidade Estadual do Sudoeste da Bahia/UESB.

ORCID: 0000-0002-7328-6025

Recebido em: 06/09/2020

Aprovado em: 23/09/2020

\section{Elieusa e Silva Sampaio}

Professora Adjunta III da Escola de Enfermagem da Universidade Federal da Bahia. Doutora em Medicina e Saúde. Mestra em Enfermagem. Especialista em Enfermagem Intensivista. Escola de Enfermagem/UFBA. ORCID: 0000-0003-1702-7296

INTRODUÇÃO

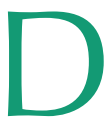
estacado como uma das principais causas de mortalidade na população brasileira(1), o Acidente Vascular Cerebral (AVC) é alvo de uma das linhas de cuidado prioritárias da Rede de Atenção às Urgências e Emergências (RUE). A preocupação com a doença é refletida tanto por sua incidência como pelo seu potencial em cau- sar danos neurológicos, influenciando a qualidade de vida da pessoa acometida, de sua família e cuidadores.

Conforme Relatório de Gestão da Secretaria de Atenção à Saúde do Ministério da Saúde, em 2012 foram constatados 126.805 mil óbitos por AVC que desencadeou medidas para ampliação dos leitos do Sistema Único de Saúde para esses pacientes. Atualmente existem 57 hospitais habilitados no atendimento especializado ao AVC (2).

Em estudo de base nacional, o Nordeste foi referido como a região brasileira com mais casos da doença em pessoas de 30 a $59 \operatorname{anos}^{(3)}$, o que demonstra a precocidade da doença e confirma a necessidade de estabelecer processos de ava- 
liação e adesão às ações de saúde, como medidas de prevenção às complicações e redução da morbimortalidade.

A Linha de Cuidado do AVC, através de ações propostas aos componentes da RUE, tem como objetivo reduzir a morbimortalidade, a partir de estratégias de disseminação do conhecimento sobre a doença; melhoria da resolutividade da rede básica de atenção no aspecto do controle adequado dos fatores de risco para doenças vasculares; qualificação do Serviço de Atendimento Móvel de Urgência (SAMU) para atendimento e referencia adequado do paciente; credenciamento de serviços de atendimento de urgência com capacidade para realização do atendimento geral e terapia trombolítica; ampliação dos leitos hospitalares para reabilitação e cuidados crônicos complexos e instituição de retaguarda ambulatorial para a assistência sequencial após a alta hospitalar. ${ }^{(4)}$

As sequelas para as pessoas que desenvolvem o AVC estão relacionadas ao acesso ao serviço de saúde, cuja melhoria tende a reduzir as incapacidades e morte. Os tratamentos atuais com trombolítico (medicamentoso) ou trombectomia (cirúrgico) refletem em melhores resultados na recuperação funcional do paciente, mas o acesso às unidades que os realizam deve ocorrer em tempo oportuno. ${ }^{(5)}$ No entanto, existem fatores que contribuem para a demora do acesso ao serviço especializado adequado refletindo em piores resultados em saúde e qualidade de vida.

Desta maneira, o objetivo deste estudo se constitui em analisar os fatores que retardam o atendimento dos pacientes na fase aguda do AVC em um hospital público de referência. Os resultados podem contribuir para embasar protocolos de assistência, avaliar a necessidade de capacitar profissionais e de realizar estratégias para esclarecer a população aspectos da doença que fortaleçam a identificação desta e permitam que a assistência seja realizada em tempo adequado.

\section{MÉTODOS}

Trata-se de estudo transversal, realizado em Unidade de Referência em AVC de um hospital público na Bahia, visando atender ao Protocolo Clínico e Diretrizes Terapêuticas para a Trombólise no Acidente Vascular Cerebral Isquêmico pelo SUS, instituído pelo Ministério da Saúde. ${ }^{(6)}$

A amostra foi constituída por 56 pacientes diagnosticados com AVC que deram entrada na Unidade de Referência (UR). Os critérios de inclusão foram pacientes com AVC isquêmico admitidos na UR com início do evento menor que cinco dias, para as respostas fossem as mais fidedignas possíveis. Houve exclusão de pacientes com AVC hemorrágico, sem condições de responder aos questionamentos e sem familiares responsáveis. Após aplicação dos critérios de inclusão e de exclusão, as entrevistas foram aplicadas à 50 pacientes e/ou a familiares.

Os dados foram coletados no período de abril a julho de 2014. Foram coletadas em prontuário informações sobre a clínica dos pacientes, tempos de chegada e de atendimento no hospital e sobre a realização de trombólise. O questionário aplicado continha: idade, sexo, data do evento e horário em que foram percebidos os sintomas, se o paciente estava sozinho na hora do evento, se houve companhia de familiar ou de outra pessoa até o hospital, se o paciente morava sozinho, qual foi o meio de transporte utilizado até o hospital, tempo do início dos sintomas até a chegada no serviço especializado, se realizou tomografia, se houve dificuldades para chegar ao hospital e se procurou outro serviço antes de ser encaminhado ao hospital referência.

Para ter a garantia de resposta ao questionário, a coleta de dados foi realizada através de visitas esporádicas durante à tarde, período em que os familiares estariam presentes, conforme o horário da visita estabelecido pela Unidade.

Os dados foram tabulados mediante a utilização do programa Statistical Package for the Social Sciences (SPSS) versão 21. Na estatística descritiva, foi realizada a análise e interpretação dos dados através das distribuições de frequências absolutas e relativas. Foi utilizado teste Qui-Quadrado para Tempo Transcorrido do Evento até a Unidade Especializada e Sexo e Tempo Transcorrido do Evento até o hospital e Trombólise. O nível de significância foi de $5 \%$.

$\mathrm{O}$ projeto de pesquisa foi submetido ao Comitê de Ética da Escola de Enfermagem da Universidade Federal da Bahia e foi aprovado sob parecer número 528.578 e CAAE 25203113.1.0000.5531, atendendo as exigências éticas da pesquisa conforme Resolução $n^{\circ} 466 / 2012^{(7)}$. Todos os sujeitos confirmaram o aceite de sua participação através da assinatura do Termo de Consentimento Livre e Esclarecido (TCLE) antes da coleta de dados.

\section{RESULTADOS}

Foram entrevistadas 50 pessoas com diagnóstico de AVC internadas na Unidade de Referência. A média de idade foi de 61,2 ( \pm 13 anos), com idade mínima de 28 anos e a idade máxima de 83 anos. As características dos pacientes com AVC e acesso à unidade de referência hospitalar são apresentadas na Tabela 1.
Tabela 1 - Características dos pacientes com AVC e acesso à unidade de referência hospitalar, Bahia, Brasil, 2014.

\begin{tabular}{lll}
$\begin{array}{l}\text { Características dos pacientes com AVC e acesso à unidade } \\
\text { de referência hospitalar }\end{array}$ & $\mathbf{n}$ & $\%$ \\
Sexo & 29 & 58 \\
\hline Masculino & 21 & 42
\end{tabular}

Familiar presente no momento do AVC 


\begin{tabular}{lll}
\hline Sim & 37 & 74 \\
\hline Não & 13 & 26 \\
\hline Paciente vive sozinho & & \\
\hline Sim & 06 & 12 \\
\hline Não & 44 & 88 \\
\hline Quem acompanhou o paciente & & \\
\hline Familiar & 43 & 86 \\
\hline Sem acompanhante & 04 & 8 \\
\hline Vizinho & 03 & 6 \\
\hline Turnos & & \\
\hline Manhã (01-12:55h) & 32 & 64 \\
\hline Tarde (13-17:55h) & 12 & 24 \\
\hline Noite (18-24:55h) & 06 & 12 \\
\hline Meio de Transporte & & \\
\hline Carro próprio & 25 & 50 \\
\hline SAMU & 10 & 20 \\
\hline Outros & 07 & 14 \\
\hline Táxi & 05 & 10 \\
\hline Ônibus & 02 & 04 \\
\hline Andando & 01 & 02 \\
\hline Fonte: dados da pesquisa. & & \\
\hline
\end{tabular}

Tabela 2. Tempo Transcorrido do AVC até a Unidade Especializada e Sexo. Salvador. 2014

\begin{tabular}{lccc} 
Tempo & \multicolumn{3}{c}{ Sexo } \\
Masculino & Feminino & P \\
$<1$ hora & 0 & 1 & \\
\hline $1-2$ horas & 0 & 2 & \\
\hline $2-3$ horas & 5 & 3 & \\
\hline $3-4$ horas & 2 & 6 & \\
$4-5$ horas & 3 & 1 & \\
\hline 5 horas & 19 & 8 & \\
\hline Total & 29 & 21 & \\
\hline
\end{tabular}

Fonte: dados da pesquisa.

Dentre os outros meios de transporte, pacientes foram transferidos de ambulância inter-hospitalar (2\%), por ambulância hospitalar intermunicipal $(4 \%)$, por ambulância de serviço particular (2\%), ambulância vinculada à Unidade de Pronto Atendimento (UPA) (2\%). Houve ainda um paciente que já estava internado no hospital para realização de procedimento cirúrgico e no primeiro dia de pós-operatório apresentou AVC, sendo apenas transferido da enfermaria para a unidade de AVC ( $1 \%)$.

O tempo médio entre o início dos sintomas e a chegada à emergência hospitalar foi de 12 horas e 27 minutos, sendo que $22 \%$ dos pacientes chegaram nas primeiras 3 horas e $16 \%$ dos pacientes chegaram entre 3 e 4 horas do início dos sintomas, $8 \%$ chegaram entre 4 e 5 horas e $54 \%$ chegaram após $5 \mathrm{~h}$ do ictus.

Em relação ao tempo transcorrido do evento até a Unidade Especializada e sexo, as mulheres chegaram mais rápido ao hospital quando comparadas com os homens, mas esta diferença não foi estatisticamente significante $(p=0,076)$ (Tabela 2), entretanto, a associação de pacientes que fizeram uso do trombolítico venoso e sexo, demonstrou que quem mais realizou este procedimento foram as mulheres $(20 \%)$ em relação aos homens $(12 \%)$ e esta diferença foi estatisticamente significante $(p=0,044)$.

Dos pacientes, $40 \%$ procuraram outros serviços antes, percorrendo cinco caminhos diferentes. Seis pessoas procuraram hospitais que não tinham a estrutura adequada para os pacientes de AVC; duas pessoas procuraram Unidades Básicas de Saúde; três pessoas procuraram hospitais na região metropolitana (cidades próximas a Salvador); seis pessoas procuraram hospitais nas cidades do interior do Estado da Bahia, que não tiveram recursos para atendê-los; e nove pessoas se dirigiram à Unidades de Pronto Atendimento. Os pacientes que buscaram o Hospital Referência como primeira escolha, foram os que chegaram mais rápido ao hospital e realizaram a terapia trombolítica.

Todos os pacientes que participaram da pesquisa realizaram o exame de tomografia computadorizada de crânio para elucidar o diagnóstico, e 32\% (16) foram submetidos ao procedimento da trombólise.

Quanto ao transporte até o hospital, os pacientes, independente do sexo, se comportaram de forma similar, utilizando preferencialmente o carro próprio, seguido do SAMU. A associação entre transporte e sexo não foi estatisticamente significante $(p=0,450)$.

A associação entre o tempo transcorrido do AVC até o hospital e realização da trombólise, demonstrou que pacientes que chegaram até 4 horas no hospital re- 
ferência fizeram uso da trombólise e essa diferença foi estatisticamente significante $(p=0,000)$ (Tabela 3).

Apenas $9(18 \%)$ pacientes referiram dificuldades para chegar ao hospital. Tais entraves foram agrupadas em dificuldades com o transporte, incluindo o trânsito engarrafado (3), demora do ônibus (1), realizar transporte intermunicipal de carro próprio (1); dificuldades com o acompanhante: o fato de estar só para pegar condução (1) ou demora em conseguir contato com a família (1); barreiras geográficas como morar na região metropolitana e "estar tarde" (em relação ao horário) para ir para o hospital (1); o fato de morar no interior ou na região metropolitana de Salvador (4); entraves burocráticos e do serviço (1); e ter que aguardar a disponibilidade da ambulância inter-hospitalar para realizar o transporte (1). Além desses, foram citados os entraves relacionados ao desconhecimento sobre a doença (1) ou o desconhecimento da existência de uma unidade especializada para atendimento ao AVC (1).

Quanto ao atendimento, o atraso foi atribuído à demora do SAMU em regular o paciente para o hospital, apesar da equipe do SAMU ter chegado rapidamente (2); demora na confecção de ficha no Hospital Referência (2); um deles informou que a demora ainda foi maior pelo fato da impressora da recepção estar quebrada; e que houve demora de atendimento pelo médico na emergência hospitalar (1).

\section{DISCUSSÃO}

No estudo houve predomínio de pacientes do sexo masculino e média de idade de 61,3 anos. Esses dados corroboram com outros estudos realizados no Brasil, como em Minas Gerais que apontou serem $55 \%$ homens e média de idade de 64,3 anos ${ }^{(8)}$, em Santa Catarina com 51\% de homens e média de idade de 66,2 anos. ${ }^{(9)}$ Já na Holanda, cerca de metade (50,2\%) dos pacientes eram homens, com média de idade de 69,4 anos. ${ }^{(10)}$

A identificação de pacientes jovens com AVC está se tornando cada vez mais comum devido a diversas características culturais e modos de vida da população que refletem em aumento dos fatores de riscos para a doença, com destaque para a hipertensão arterial, tabagismo, sedentarismo, sobrepeso e obesidade, desequilíbrio nutricional e a dislipidemia.(11)

Foi evidenciado neste estudo que as mulheres com AVC chegaram mais rápido do que os homens e foram trombolizadas em número maior. As questões relacionadas ao gênero podem ser obstáculos à busca do serviço de saúde pelo homem, devido sentimentos como medo e vergonha, mas também pelo comportamento de descuido e atribuição de ${ }^{\circ}$ utras prioridades que minimizam as preocupações com a saúde. ${ }^{(12)}$

O predomínio do período da manhã na identificação dos casos de AVC pode ter relação com o denominado 'Wake-

Tabela 3: Tempo Transcorrido do AVC até o hospital e Trombólise. Salvador. 2014

\begin{tabular}{|c|c|c|c|}
\hline \multirow{2}{*}{ Tempo } & \multicolumn{2}{|c|}{ Realização de Trombólise } & \multirow{2}{*}{$\mathbf{p}$} \\
\hline & $\operatorname{Sim}(n)$ & Não(n) & \\
\hline$<1$ hora & 0 & 1 & \multirow{7}{*}{$P=0,000$} \\
\hline $1-2$ horas & 2 & 0 & \\
\hline 2-3 horas & 5 & 3 & \\
\hline 3-4 horas & 8 & 0 & \\
\hline 4-5 horas & 1 & 3 & \\
\hline$>5$ horas & 0 & 27 & \\
\hline Total & 16 & 34 & \\
\hline
\end{tabular}

Fonte: dados da pesquisa. -up', que se refere ao fato do paciente acordar com os sintomas. Estudo demonstra que $25 \%$ dos pacientes com AVC perceberem alterações neste período. Essa característica pode estar relacionada com a gravidade da doença e pode ter relação direta com o diabetes e o estilo de vida sedentário. O 'Wake-up', ainda, está intrinsicamente relacionado à perda de janela terapêutica devido à dificuldade em precisar o início dos sintomas. ${ }^{(13)}$

Neste estudo, o tempo médio superior a 12 horas para chegar ao hospital é muito superior ao tempo máximo de 210 minutos esperado para ser gasto no pré-hospitalar, incluindo o tempo de decisão e deslocamento. Isso difere de estudos em países europeus como na Espanha em que pacientes chegam à emergência com mediana de tempo de 138 minutos ${ }^{(14)}$ ou na Holanda, em que o tempo médio entre o início dos sintomas e o tempo de chegada ao pronto-socorro foi de $240 \mathrm{~min} .{ }^{(10)}$

As condições socioeconômicas e o acesso aos serviços de saúde são apontados como fatores que influenciam atrasos pré-hospitalares. ${ }^{(15,16)} \mathrm{O}$ não reconhecimento dos sinais e sintomas é outro motivo de atraso do tratamento e reconhecê- los pode ser um motivo para não acionar o serviço pré-hospitalar. ${ }^{(17)}$

Foi observado que uma parcela dos pacientes que procurou atendimento em outro serviço de saúde anteriormente, não conseguiu chegar dentro da janela terapêutica. A complexidade do sistema de saúde e suas rotinas burocratizadas podem limitar a dinamicidade do cuidado em saúde ${ }^{(18)}$ e colaborar para as barreiras aos serviços especializados.

Neste estudo foram destacados cinco tipos de unidades de saúde que estiveram envolvidas no itinerário do paciente antes da chegada ao hospital de referência. Os caminhos pré-hospitalares até a chegada ao hospital são numerosos, complexos e que refletem em atrasos, dependendo da porta de entrada utilizada. Conforme estudo na Holanda, paciente percorreram 10 caminhos diferentes para chegar à Unidade de AVC, sendo que apenas 5\% tiveram 
contato com apenas um serviço de saúde antes de chegar ao Hospital. ${ }^{(10)}$

Os pacientes que buscaram o Hospital de Referência como primeira escolha, foram os que tiveram horários de chegada em tempo mais oportuno para a terapia trombolítica. Em contrapartida, estar em cidades do interior constitui atraso na chegada ao hospital e inelegibilidade de trombólise.

A deficiência de conhecimento sobre os sintomas e necessidade de atendimento precoce no AVC e outras dificuldades como transportes e busca de serviço médico anterior retardam o atendimento adequado ao paciente. ${ }^{(19)}$

As recomendações atuais apontam para a organização de sistemas integrados de atenção ao AVC incluindo o acionamento dos serviços de saúde pré-hospitalares. Isso envolve a necessidade de outros serviços terciários contra referenciarem pacientes em janela terapêutica para centros especializados em AVC em tempo oportuno. ${ }^{(20)}$

Em relação ao meio de transporte, o carro próprio foi o mais utilizado, seguido do SAMU. Porém, utilizar alguns transportes é fator de atraso no atendimento dos pacientes. O carro próprio esteve associado ao maior número de pacientes com atraso no atendimento e o atendimento e transporte pelo SAMU associado à chegada à unidade especializada com intervalo de tempo inferior a 5 horas.

Apenas um paciente que utilizou outro tipo de ambulância, que não o SAMU, foi submetido à trombólise, após identificação do AVC em outro hospital e encaminhamento para a unidade especializada. $\mathrm{O}$ acesso do paciente à Unidades de Referências em AVC, quando precoce, relaciona-se a uma melhor recuperação e à redução de sequelas graves. ${ }^{(21)}$

Assim como no presente estudo, em pesquisa realizada na Argentina apontou que sete em cada dez pessoas atendidas não usaram o serviço pré-hospitalar, indo ao pronto-socorro em transporte próprio ou em veículos alugados. Foi percebido que dificuldades físicas para utilizar seu próprio transporte fez os pacientes acio- narem o serviço pré-hospitalar, mas não houve benefício de tempo para quem utilizou esse serviço. ${ }^{(17)}$

Já na Espanha, a utilização do serviço pré-hospitalar melhorou o tempo de atendimento tendo em vista média de apenas 15 minutos no atendimento ao paciente, antes de se deslocar para o hospital. (14) Em Rhône, na França, esse intervalo de tempo foi de 14 minutos. ${ }^{(22)}$ No presente estudo, fatores como longo tempo no aguardo da regulação médica para permissão de deslocamento para o Hospital Referência pode significar atraso no atendimento pré-hospitalar.

A notificação do hospital pelo serviço pré-hospitalar antes da chegada do paciente foi associada a um melhor tempo médio de porta-imagem e de porta-aguIha. Não notificar, no entanto, não encurta os tempos hospitalares frente a um código de AVC. ${ }^{(23)}$

Para os pacientes que vivem sozinhos, não foi observado atraso no tempo de chegada ao serviço especializado. Mesmo vivendo sozinhos, a grande maioria das pessoas que acompanharam os vitimados pelo AVC foi um familiar, o que demonstra a importância da rede de apoio da família e sua influência na coesão e adaptabilidade familiar (24).

É importante frisar que apesar da porcentagem de pacientes trombolisados neste estudo ser de $32 \%$, essa porcentagem não significa a relação entre os pacientes que procuraram o serviço de saúde e foram trombolisados. Os pacientes incluídos na pesquisa já estavam internados na Unidade de AVC. Na Holanda, em toda a população de AVC, o tratamento trombolítico pode ser administrado apenas a uma minoria entre 1 e $8 \%{ }^{(10)}$ e na Argentina a média de trombólise da cidade é de $1 \%$. ${ }^{(17)}$

A realização do exame de tomografia computadorizada para todos os pacientes da pesquisa pode estar associada ao fato deste exame ser requisito para uma avaliação neurológica e admissão na unidade especializada.

Apenas 9 pacientes referiram dificuldades para chegar ao hospital referência, mesmo que somente poucos tenham sido trombolisados. Isso perpassa pela não identificação dos riscos, da gravidade da doença e não conhecimento sobre o tempo necessário para tratamento oportuno. Há necessidade de fortalecer a educação comunitária sobre a doença, em especial para os pacientes com fatores de risco, com divulgação dos sistemas de acionamento de serviço de emergência; organização dos serviços de saúde; e informação sobre a realização de terapia trombolítica até 4,5h. ${ }^{(20)}$

Existem fatores contextuais, cognitivos e comportamentais que reduzem o tempo de atraso pré-hospitalar e o tempo até o tratamento como pedir ajuda imediatamente após o início dos sintomas, apresentar os sintomas durante o dia ou no fim de semana, apresentar os sintomas fora de casa, perceber que não controla os sintomas sem assistência, reconhecer e interpretar o AVC como emergência médica, ligar para $\mathrm{o}$ atendimento pré-hospitalar como primeiro atendimento médico, ter dificuldade na fala por ser um sintoma mais alarmante. ${ }^{(14)}$

No ambiente hospitalar, por sua vez, alguns pacientes e familiares referiram demora para fazer a ficha de atendimento na emergência do hospital e demora para passar pelo médico neurologista da emergência. Pacientes avaliados por uma equipe multidisciplinar na imediata chegada da ambulância à Emergência e encaminhamento rápido ao exame de tomografia aumenta a porcentagem de pacientes submetidos à trombólise. ${ }^{(25)}$ Dessa maneira, são importantes estudos futuros que investiguem a efetividade hospitalar.

\section{CONCLUSÃO}

Diversos fatores retardam o atendimento dos pacientes na fase aguda do AVC em um hospital público de referência, apesar dos pacientes e familiares identificarem de forma limitada estes fatores. Pode-se atribuir a este fato a não consciência sobre as consequências da doença e trazer à tona a importância da educação em saúde direcionada à população a fim 
de que esta identifique, acione o Serviço de Atendimento Móvel de Urgência ou encaminhe este paciente de forma rápida e segura à Unidade de Referência.

Os fatores de atraso, vinculados às instituições, e não apenas à decisão do paciente, devem ser observados e minorados a fim de que correções sejam feitas e mais pacientes sejam submetidos ao tratamento preconizado. Protocolos de assistência, sensibilização e educação permanente dos profissionais e elaboração de estratégias no serviço devem permitir que a assistência seja realizada em tempo oportuno.

Desta maneira, a RUE deve ser estruturada conforme a Linha de Cuidados em
Acidente Vascular Cerebral para permitir que a regulação em situações de emergência ocorra de forma mais efetiva, o que inclui a capacidade de respostas às necessidades das instituições de menor complexidade, além da capacitação dos profissionais no reconhecimento das manifestações da doença.

\section{Referências}

1. Araújo JP, Darcis JVV, Tomas ACV, Mello WA. Tendência da Mortalidade por Acidente Vascular Cerebral no Município de Maringá, Paraná entre os Anos de 2005 a 2015. Int. J. Cardiovasc. Sci. [Internet]. 2018 [acesso: 12/07/2020]; 31 (1): 56-62. doi: 10.5935/2359-4802.20170097.

2. Brasil. Ministério da Saúde. Secretaria de Atenção à Saúde. Relatório de Gestão 2017. Brasilia: MS; 2018. Disponível em: https://www.gov.br/saude/ptbr/centrais-de-conteudo/relatorio-de-gestao-2017-completo-para-ms-pdf/view 3. Schmidt MH, Selau CM, Soares PS, Franchi EF, Piber VD, Quatrin LB. Acidente vascular cerebral e diferentes limitações: uma análise interdisciplinar. Arq. Cienc. Saúde UNIPAR, Umuarama. 2019 [acesso: 11/08/2020]; 23 (2): 139-144. doi: 10.25110/arqsaude.v23i2.2019.6404

4. Brasil. Ministério da Saúde. Linha de Cuidado do Acidente Vascular Cerebral AVC na Rede de Atenção às Urgências. Brasilia: MS; 2013. Disponivel em: http:// bvsms.saude.gov.br/bvs/saudelegis/gm/2012/PRT0665_12_04_2012.html. 5. Alamri y, Zafar A. Changing time for acute stroke management: What comes after dawn? Neurosciences (Riyadh). 2019 [acesso: 12/07/2020]; 24 (2): 142. doi: 10.17712/nsj.2019.2.20180038

6. Brasil. Ministério da Saúde. Portaria n. 664, de 12 de abril de 2012. Aprova o Protocolo Clínico e Diretrizes Terapêuticas - Trombólise no Acidente Vascular Cerebral Isquêmico Agudo. Disponível em: http://bvsms.saude.gov.br/bvs/saudelegis/gm/2012/PRT0664_12_04_2012.html

7. BRASIL. Ministério da Saúde. CNS. Resolução n466, de 12 de dezembro de 2012. Disponivel em: https://goo.gl/zH8isE. Acesso em: 02 jun. 2018

8. Mourão AM, Vicente LCC, Chaves TS, Sant'Anna RV, Meira FC, Xavier RMB, Tanure MTA, et al. Perfil dos pacientes com diagnóstico de AVC atendidos em um hospital de Minas Gerais credenciado na linha de cuidados. Revi. Bras. Neurol. 2017 [acesso: 11/08/2020]; 53 (4): 12-16. Disponível em: http://docs.bvsalud. org/biblioref/2017/12/876884/rbn-534-2-perfil-dos-pacientes-com-diagnostico-de.pdf

9. Barella RP, Duran VAA, Pires AJ, Duarte RO. Perfil do atendimento de pacientes com acidente vascular cerebral em um hospital filantrópico do sul de Santa Catarina e estudo de viabilidade para implantação da unidade de AVC. Arq. Catarin Med. 2019 [acesso: 11/08/2020]; 48 (1): 131-143. Disponível em: http://www. acm.org.br/acm/seer/index.php/arquivos/article/view/432/334

10. Doggen CM, Zwerink M, Droste HM, Brouwers PJAM, Houwelingen GK, Eenennaam FL, Egberink RE. Prehospital paths and hospital arrival time of patients with acute coronary syndrome or stroke, a prospective observational study. BMC Emerg. Med. 2016 [acesso: 12/08/2020]; 16 (3). doi: 10.1186/s12873-015-0065-y 11. Correia JP, Figueiredo AS, Costa HM, Barros P, Veloso LM. Investigação Etiológica do Acidente Vascular Cerebral no Adulto Jovem. Medicina Interna [Internet]. 2018 [acesso: 25/07/2020]; 25 (3): 213-223. Disponível em: http://www.scielo.mec.pt/scielo.php?script=sci_arttext\&pid=S0872-671X2018000300012\&lng=pt. http://dx.doi.org/10.24950/rspmi/ revisao/200/3/2018.

12. Teixeira DBS, Silvana PLC. Atenção à saúde do homem: análise da sua resistência na procura dos serviços de saúde. Rev. Cuba. Enferm., 2016 [acesso: 25/07/2020]; 32(4):1561-2961. Disponível em: http://www.revenfermeria.sld.cu/ index.php/enf/article/view/985.

13. Diniz DLO, Barreto PR, Bruin PFC, Bruin VMS. Wake-up stroke: Clinical charac teristics, sedentary lifestyle, and daytime sleepiness. Rev. Assoc. Med. Bras. [Internet]. 2016 [acesso: 25/07/2020]; 62 (7): 628-634. Disponível em: http://www.
scielo.br/scielo.php?script=sci_arttext\&pid=S0104-42302016000700628\& $1-$ ng=en. https://doi.org/10.1590/1806-9282.62.07.628.

14. Soto-Cámara R, González-Santos J, González-Bernal J, Martín-Santidrian A, Cubo E, Trejo-Gabriel-Galán JM. Factors Associated with Shortening of Prehospital Delay among Patients with Acute Ischemic Stroke. J Clin Med. 2019 [citado 27 Jul 2020]; 8 (10): 1712. Disponivel em: https://www.ncbi.nlm.nih.gov/pmc/ articles/PMC6832968/ DOI: 10.3390/jcm8101712

15. Bray BD, Paley L, Hoffman A, James M, Gompertz P, Wolfe CDA, et al. Socioeconomic disparities in first stroke incidence, quality of care, and survival: a nationwide registry-based cohort study of 44 million adults in England. Lancet Public Health. 2018 [citado 27 Jul 2020]; 3 (4): e185-e193. doi: 10.1016/S24682667(18)30030-6.

16. Niklasson A, Herlitz J, Jood K. Socioeconomic disparities in prehospital stroke care. Scand J Trauma Resusc Emerg Med. 2019 [citado 29 Jul 2020]; 27 (1): 53. Disponivel em: https://pubmed.ncbi.nlm.nih.gov/31046804/ DOl: 10.1186/ s13049-019-0630-6

17. Hawkes MA, Farez Mauricio F, Calandri IL,Ameriso Sebastián F . Perception of stroke symptoms and utilization of emergency medical services. Arq. Neuro-Psiquiatr., São Paulo. 2016 [citado 29 Jul 2020]; 74(11):869-74. doi: 10.1590/0004-282x20160142.

18. Arruda C, Lopes SGR, Koerich MHAL, Winck SR, Meirelles BHS, Mello ALSF. Redes de atenção à saúde sob a luz da teoria da complexidade. Esc. Anna Nery [Internet]. 2015 [citado 29 Jul 2020]; 19 (1): 169-173. doi: https://doi. org/10.5935/1414-8145.20150023.

19. Fonseca LHO, Rosa MLG, Silva AC, Maciel RM, Volschan A, Mesquita ET. Análise das barreiras à utilização de trombolíticos em casos de acidente vascular cerebral isquêmico em um hospital privado do Rio de Janeiro, Brasil. Cad. Saúde Pública, Rio de Janeiro. 2013 [citado 05/08/2020]; 29 (12): 2487-2496.

20. Harris DR. A new era in stroke care. CJEM. 2018 [citado 05/08/2020]; 20 (5): 655-657. doi: 10.1017/cem.2018.448.

21. Langhorne P, O'Donnell MJ, Chin SL, Zhang H, Xavier D, Avezum A, et al. Practice patterns and outcomes after stroke across countries at different economic levels (INTERSTROKE): an international observational study. Lancet. 2018 [citado 11/08/2020]; 391 (10134): 2019-2027. doi: 10.1016/S0140-6736(18)30802-X

22. Freyssenge J, Renard F, Schott AM, Nighoghossian N, Tazarourte K, Khoury CEI. Measurement of the potential geographic accessibility from call to definitive care for patient with acute stroke. Int J Health Geogr. 2018 [citado 11/07/2020]; 17:1. doi: 10.1186/s12942-018-0121-4

23. Kim DH, Nah HW, Park HS, Choi JH, Kang MJ, Huh JT, Cha JK. Impact of Prehospital Intervention on Delay Time to Thrombolytic Therapy in a Stroke Center with a Systemized Stroke Code Program. J. Stroke Cerebrovas. Dis. 2016 [citado 11/07/2020]; 25 (7): 1665-1670. doi: 0.1016/j.jstrokecerebrovasdis.2016.02.011 24. Seibel BL, Falceto OG, Hollist CS, Springer P, Fernandes CLC, Koller SH. Rede de apoio social e funcionamento familiar: estudo longitudinal sobre famílias em vulnerabilidade social. Pensando fam. 2017[citado 11/07/2020]; 21 (1): 120-136. Disponível em http://pepsic.bvsalud.org/scielo.php?script=sci_arttext\&pid=S1679-494X2017000100010\&lng=pt\&tlng=pt.

25. Madhok DY, Keenan KJ, Cole SB, Martin C, Hemphil JC. Prehospital and Emergency Departament Focused Mission Protocol Improves Thrombolysis Metrics for Suspected Acute Stroke Patients. J. Stroke Cerebrovasc. Dis. 2019 [citado 21/08/2020]; 28 (12). doi: 10.1016/j.jstrokecerebrovasdis.2019.104423. 